\title{
Morphogenesis of Magnetite Mesocrystals: Interplay between Nano- particle Morphology and Solvation Shell
}

\author{
Julian Brunner ${ }^{1}$, Britta Maier ${ }^{1}$, Sabrina L.J. Thomä ${ }^{2}$, Felizitas Kirner ${ }^{1}$, Igor A. Baburin ${ }^{3}$, Dmitry Lap- \\ $\mathrm{kin}^{4}$, Rose Rosenberg ${ }^{1}$, Sebastian Sturm ${ }^{5}$, Dameli Assalauova ${ }^{4}$, Jerome Carnis ${ }^{4}$, Young Yong Kim ${ }^{4}$, Zhe \\ Ren $^{4}$, Fabian Westermeier ${ }^{4}$, Sebastian Theiss ${ }^{6}$, Horst Borrmann ${ }^{7}$, Sebastian Polarz ${ }^{6}$, Alexander Ey- \\ chmüller $^{3}$, Axel Lubk ${ }^{4}$, Ivan A. Vartanyants ${ }^{4,8}$, Helmut Cölfen ${ }^{1}$, Mirijam Zobel ${ }^{2}$, and Elena V. Sturm ${ }^{1 *}$. \\ ${ }^{1}$ Department of Chemistry, Universität Konstanz, Universitätsstraße 10, 78467 Konstanz, Germany. E-mail: \\ elena.sturm@uni-konstanz.de \\ 2 Department of Chemistry, University of Bayreuth, Universitätsstraße 30, 95447 Bayreuth, Germany \\ ${ }^{3}$ Department of Chemistry, TU Dresden, Bergstraße 66b, 01062 Dresden, Germany \\ ${ }^{4}$ Deutsches Elektronen-Synchrotron DESY, Notkestrasse 85, D-22607 Hamburg, Germany \\ ${ }^{5}$ Advanced Methods of Electron Microscopy (AMEM), Leibniz Institute for Solid State and Materials Research (IFW) Dres- \\ den, Helmholtzstraße 20, 01069 Dresden, Germany \\ ${ }^{6}$ Institute of Inorganic Chemistry, Leibniz-University Hannover Callinstrasse 9, 30167 Hannover, Germany \\ ${ }^{7}$ Max-Planck-Institute for Chemical Physics of Solids, Nöthnitzer Straße 40. 01187 Dresden, Germany). \\ ${ }^{8}$ National Research Nuclear University MEPhI (Moscow Engineering Physics Institute), Kashirskoe shosse 31, Moscow \\ 115409, Russian Federation
}

KEYWORDS: mesocrystals, nanoparticles, iron oxide, morphogenesis, solvation shell.

\begin{abstract}
Nanoparticle assemblies with long range packing order and defined crystallographic orientation of building blocks, i.e., mesocrystals, are of high interest not only because of their unique physical properties, but also due to their complex structure and morphogenesis. In this study, faceted mesocrystals have been assembled from the dispersion of truncated cubic-shaped iron oxide nanoparticles stabilized by oleic acid (OA) molecules using the non-solvent "gas phase diffusion technique" into an organic solvent. The effects of synthesis conditions as well as of the nanoparticle size and shape on the structure and morphogenesis of mesocrystals were examined. The interactions of OA capped iron oxide nanoparticles with solvent molecules were probed by analytical ultracentrifugation and double difference pair distribution function analysis. It was shown that the structure of the organic shell significantly depends on the nature and polarity of solvent molecules. For the nonpolar solvents, the interaction of the aliphatic chains of OA molecules with the solvent molecules is favorable and the chains extend into the solvent. The solvation shell around the nanoparticles is more extended in nonpolar and more compact in polar solvents. The interplay between the shape of the iron oxide nanocrystals and the specific interaction of nanoparticles with solvent molecules governs the process of self-assembly towards mesocrystals and determines the crystal structure and the related morphology of mesocrystals. The observed changes in packing symmetry are to a certain extent in analogy to structural polymorphism known for "classical" (atomic and molecular) crystals.
\end{abstract}

\section{INTRODUCTION}

The synthesis of nanocrystals and their self-assembly to superstructures is of major importance in current materials science ${ }^{1-}$ ${ }^{3}$, because nanocrystals frequently exhibit exceptional size-dependent properties in comparison to their bulk material. ${ }^{4-6}$ The nanoparticle assemblies not only maintain some of these sizedependent properties, they can additionally exhibit collective properties resulting from the interaction of nanoparticles. ${ }^{7-11}$ Consequently, assembly of nanocrystals into macroscopic solid superstructures are of high scientific relevance in the field of materials science and application. ${ }^{12}$
Ordered assemblies of nanocrystals have been denoted differently in the scientific literature over time, ranging from colloidal crystals, mosaic crystals, supracrystals, supercrystals, superlattices (SL) to mesocrystals. ${ }^{13-18}$ To distinguish assemblies, in which this crystallographic orientational order of the crystalline building blocks also exists, the term mesocrystals has been coined. ${ }^{19-21}$ Hence, in the most strict sense mesocrystals (socalled type I) are a special class of colloidal crystals combining a long-range order of particle packing and their preferable crystallographic orientation (i.e., atomic scale ordering). 
The assembly process of nanoparticles into ordered superstructures can be described with the so-called "non-classical" crystallization process. It is generally accepted that in contrast to the "classical crystallization" which describes the formation of crystals and/or aggregates by a sequential attachment of molecule/ions/atoms, the "non-classical crystallization" theory includes a particle-mediated crystallization process with nanoparticles acting as building blocks. ${ }^{22,}{ }^{23}$ Notwithstanding, MonteCarlo simulations and free-energy calculations enable the construction of complex phase diagrams for nanocrystal assemblies similar to classical crystals. ${ }^{24,25}$ These ordered superstructures differ from those resulting from aggregation of larger microparticles (so-called "classical colloids") and cannot be comprehensively described within the theory of colloidal stability developed by Derjaguin, Landau, Vervey, and Overbeek (so-called DLVO-theory). ${ }^{26-28}$ Indeed, at the nanometer scale, in case of small nanoparticles stabilized by surface ligands, nanoparticle interaction forces are no longer additive (so-called principle of "non-additivity"). ${ }^{29}$ Complex multiscale collective effects lead to a breakdown of DLVO theory for nanoparticles. They also include coupled dynamics of interacting nanoparticles (especially the capping organic molecules stabilizing the nanoparticles) and the surrounding media. ${ }^{29}$

In the last few years, many research groups have reported on assembly techniques of micrometer-sized mesocrystals of platinum, magnetite, lead sulfide, and silver amongst other materials. ${ }^{8,30-38}$ They have shown remarkable structures of mesocrystals, which are perfectly regular and feature well-defined facets, like "classical" crystals. Commonly, nanocrystals are assembled to mesocrystals either via "drying mediated assembly methods" or via the "gas phase diffusion technique". 8, 17, 34, 36, 37 Often, the structure of these assemblies is characterized incompletely, while the main emphasis is put on the study of explicit properties or specific applications of mesocrystals. ${ }^{10,12,39,40}$ Nevertheless, investigations with a combination of small- and wide-angle X-ray scattering techniques (SAXS and WAXS) as well as advanced microscopy techniques allow a full structural analysis of self-assembled superstructures based on nanoparticles of different composition and shape..$^{8,18,31,33,35,37,38}$ Recently developed Angular X-ray Cross-Correlation Analysis (AXCCA) method allows the determination of the superlattice distortion and relative orientation of substituting nanoparticles in mesocrystals with high precision. ${ }^{41-45}$ Similarly, great effort is put on how these crystals nucleate and grow and how the kinetics influence the non-classical crystallization of mesocrystals. ${ }^{16,37,46-51}$ Furthermore, there are many interesting results on how growth conditions can impact the final structure of mesocrystalline films. ${ }^{52-57}$ Accordingly, different superlattice structures can be obtained by solvent evaporation from dispersions with different dispersion agents as well as with nanoparticles stabilized by different ligands., ${ }^{32}$ However, an understanding of the morphogenesis of macroscopic mesocrystals is still lacking. Specifically, the exact parameters which influence the crystal structure and morphology, including packing arrangement and orientational order, remain largely unexplored.

Grazing incidence X-ray scattering is one of the widely used technique to study real-time self-assembly of nanoparticles. In situ study of $\mathrm{PbS}$ and $\mathrm{Fe}_{3} \mathrm{O}_{4}$ nanoparticle assembly by "spherical confinement" $47,58,59$ reveals that alignment of nanoparticles occurs before the superlattice densifies. It was suggested that the self-assembly process thus unfolds as a two-step process. Here, nanoparticles interact similarly to hard spheres solely by reducing the volume to the densest possible packing arrangement $(f c c)$ without solvent-mediated interactions. It was speculated that other interactions are not important and the growth of a mesocrystal depends only on the disposability of nanocrystals irrespective of the relative time of formation ${ }^{60}$. Contrary, in the case of $\mathrm{Au}$ nanoparticles, experiments and simulations show that solvent-mediated interactions can significantly affect the nucleation of colloidal crystals. ${ }^{61}$ Furthermore, it has been found that the final morphology of colloidal crystals depends on the size of the initial nanocrystals. Recently, large progress in the understanding of medium properties on nanoparticle assembly processes was made by real time tracking of growth processes of highly ordered superlattices using small-angle X-ray scattering. ${ }^{37}$ This study reveals that the assembly process of iron oxide nanoparticles is significantly affected by the evaporationdriven increase of the solvent polarity, particle concentration, and excess of surfactant ${ }^{37}$ Furthermore, theoretical considerations for nanocrystals with anisotropic shapes predict the formation of liquid-crystalline and plastic-crystalline phases with orientational and translational order, respectively. ${ }^{62}$ For superparamagnetic iron oxide nanocrystals, within the size range from $5 \mathrm{~nm}$ to $15 \mathrm{~nm}$ the magnitude of the magnetic dispersion interactions at zero-field was shown to be negligible in comparison to van der Waals forces between the nanoparticles. ${ }^{63}$

In recent years, our work focused on the preparation and structural characterization of self-assembled iron oxide mesocrystals $8,13,64-66$ prepared from highly monodisperse truncated nanocubes of iron oxide (composed of magnetite with some inclusion of maghemite (up to 20\%)) ${ }^{13}$ The morphology of these nanocrystals could be approximated as cubes slightly truncated by $\{111\},\{110\},\{310\}$ and $\{114\}$ faces. Using TEM and SAXS we analyzed the structure of self-assembled mesocrystalline films (formed from toluene dispersion by the "drying mediated" technique) and showed that the orientational order of nanocrystals within an $f c c$ superlattice is in line with the socalled "bump-to-hollow" packing principle known for molecular crystals (which aims to achieve the most efficient space filling) ${ }^{67}$ Furthermore, we confirmed that external magnetic field could significantly affect the assembly process and change the morphology of aggregates and orientational order of nanoparticles. $^{64}$

This paper intends to provide a systematic study of morphogenetic aspects of mesocrystals and represents an important step forward compared to our previous study. A detailed structural characterization of faceted mesocrystals will be discussed in a follow-up article. Herein, we took advantage of the iron oxide nanocrystals stabilized by oleic acid (OA) as building blocks to create mesocrystalline films and large-scale facetted mesocrystals using "solvent evaporation" and "gas-phase diffusion" techniques, respectively. All experiments on nanoparticles assembles were carried out without external magnetic field (i.e., in zero-field). First, we will discuss the effect of nanoparticle variables (incl. size, faceting, habit) on the crystallization process and structuring of mesocrystals. Second, we will show that the assembly process can be further influenced by the nature of solvent (tetrahydrofuran, toluene, cyclohexane, and heptane), non-solvent, and excess of surfactant. For the first time, the formation of solvation shell around oleic acid capped iron oxide nanoparticles was probed by advanced analytical techniques directly in dispersion, namely analytical ultracentrifugation 
(AUC) and double difference pair distribution function (ddPDF). The solvation shell formed in solvents with different polarity significantly influences the morphogenesis and final structure of the mesocrystals, which is also confirmed by XRD study in combination with AXCCA. Finally, we show that mesocrystals exhibit several similarities to "classical" crystals in terms of structural and morphogenetic aspects. The latter include "structural polymorphism" of mesocrystals due to different growth conditions.

\section{RESULTS AND DISCUSSION}

The nanocrystals have been synthesized as described by Disch et al. ${ }^{30}$ Six different nanocrystal batches (batch I-VI, characterized by different particle sizes and degrees of truncation of cubic nanocrystals, Table S1, Figures S1-S3). In addition to HRTEM images, the relative degree of truncation could be estimated by ratio between "equivalent edge length of cube" and "minFeret" size (Figures S2, S3). The more this value deviates from 1, the higher is the degree of truncation of the cubic particles: batch I: 0.927; batch II: 0.942; batch III: 0.940; batch IV: 0.931.These nanocrystals were assembled to mesocrystals using either an adapted approach of the "gas phase diffusion technique" (to form faceted 3D mesocrystals) or evaporation induced self-assembly (to form mesocrystalline 2D/3D films). ${ }^{13}$, 64, 68, 69 A simplified illustration of the experimental setups of the different techniques is presented in Figure 1. In the case of the gas phase diffusion technique (Figure 1 a), the destabilizing diffusion phase infiltrates the nanoparticle dispersion via the gas phase. The destabilizing diffusion phase (so-called "nonsolvent" or "poor-solvent") consists of mixture of ethanol and solvent (1:1); while tetrahydrofuran (THF), toluene, cyclohexane and heptane were used as solvents, respectively. The mesocrystals are grown on a single-crystalline silicon substrate by destabilization of a nanoparticle dispersion containing an excess of surfactant. In the case of the solvent evaporation technique (Figure $1 \mathbf{b}$ ), mesocrystals were grown directly on a TEM-grid by evaporation of the dispersion agent from the nanocrystal dispersion. a

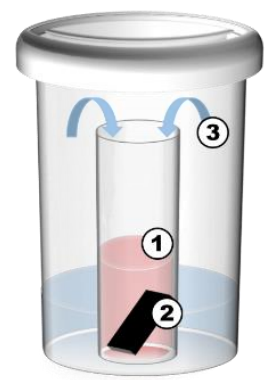

b

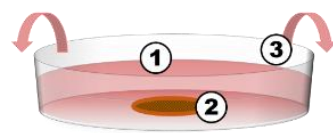

Figure 1. Schematic illustration of experimental setup. a) Mesocrystal formation by the gas phase diffusion:a glass tube with nanoparticle dispersion (1) and a vertically positioned silicon snippet (2) is stored in a glass vial containing the destabilizing diffusion phase (3). The large overall glass vial is sealed. b) Mesocrystal formation by solvent evaporation: a substrate $(2$, TEM grid) is placed within the nanocrystal dispersion (1). The dispersion agent then evaporates (3).

\section{Structural features of mesocrystals.}

The self-assembled magnetite mesocrystals from nanocrystal batch III were investigated by scanning electron microscopy (SEM), small and wide-angle X-ray diffraction methods (SAXS, WAXS), while the building blocks of these mesocrystals were analyzed with high-resolution Cs-corrected transmission electron microscopy (TEM). The collected data summarizes the major structural features of the mesocrystals (type I) with an $f c c$ superlattice symmetry $(a=21.0(3) \mathrm{nm})$ grown in cyclohexane nanoparticle dispersion (Figure 2). The morphology of these mesocrystals would be best described as trigonal truncated pyramids and is shown at different magnifications using complementary microscopy techniques (Figure $\mathbf{2}$ a, b). The HRSEM image (Figure $2 \mathbf{c}$ ) and its corresponding Fast-FourierTransformation (FFT) of the projected (111) basal face of the mesocrystal demonstrate the $p 6 \mathrm{~mm}$ plane symmetry of the packed magnetite nanocrystals with the shape of slightly truncated cubes (proven by the Cs-corrected HRTEM images along $[100]_{\mathrm{Fe} 3 \mathrm{O} 4}$ in Figure 2 d, 3 a,d,g,j). Furthermore, the SAXS pattern recorded along the [111] direction of the superlattice exhibits a typical single crystal-like pattern indicating the high long-range order of nanoparticles within the mesocrystal (Figure $2 \mathbf{e}$ ). The indexed pattern is presented in the supporting information (Figure S4). The pattern displays secondary Bragg peaks, which would be forbidden for "classical" crystals. Most probably, these signals are caused by a limited coherence of the mesocrystalline lattice. Indeed, such secondary "forbidden" Bragg peaks have already been reported for $f c c$ colloidal crystals. ${ }^{70}$ Unlike ordinary colloidal crystals, mesocrystals show typical texture-like diffraction patterns in wide-angle diffraction (Figure $2 \mathbf{f}$ ). The texture-like WAXS pattern proves the preferable orientational ordering of the nanocrystals throughout

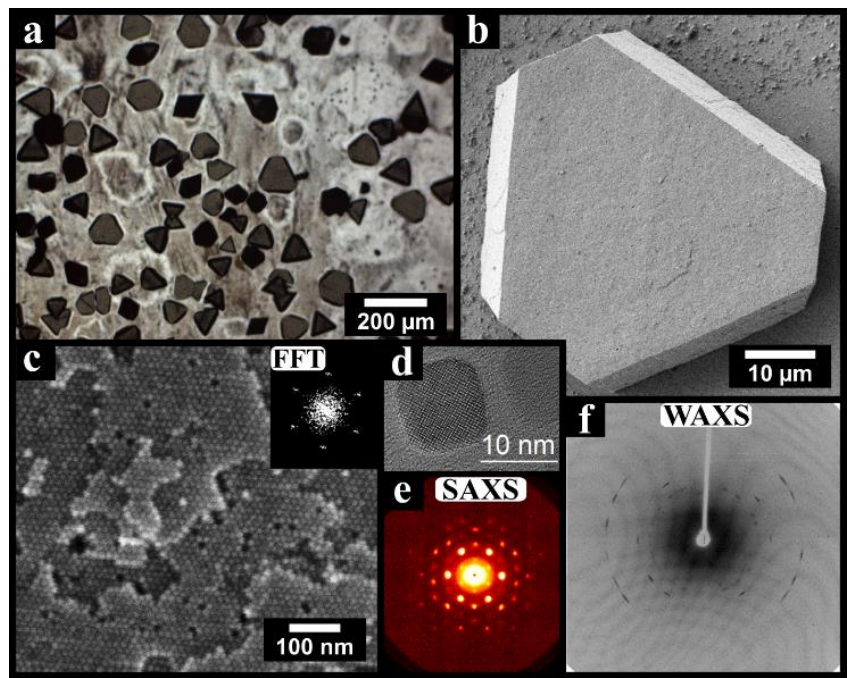

Figure 2. Summary of the major structural features of magnetite self-assembled mesocrystals (type I) with an fcc superlattice symmetry $(a=21.0(3) \mathrm{nm})$ grown in cyclohexane nanoparticle dispersion (batch III). a) Light microscopic image of mesocrystals grown on a silicon snippet. b) SEM image of a single mesocrystals with a morphology of truncated trigonal pyramid. c) HRSEM image and its corresponding FFT of the (111) basal face of the mesocrystal (shown in b) illustrating the p6mm plane symmetry of the projection of nanoparticle packing. d) HRTEM image of the magnetite nanoparticle along [100] e) Two-dimensional SAXS pattern recorded along [111] of a single mesocrystal. f) WAXS recorded from a single mesocrystal showing a typical texture-like pattern. 
the mesocrystal. Even though the data received from X-ray analysis for the structure of the mesocrystals is highly interesting, its detailed discussion is beyond the scope of this paper and will be subject of the follow-up manuscript devoted to the structural aspects of mesocrystals. Nevertheless, combining all discussed experimental evidences, we conclude that the investigated specimen can be described as a mesocrystal type $\mathrm{I}^{21}$

\section{Effect of nanoparticle shape.}

Investigations of mesocrystals formed under the same medium conditions, but with different nanocrystal batches (batch I-IV), reveal that the habit (i.e., degree of the cube truncation) of the individual building blocks significantly influences the packing symmetry of nanoparticles and mesocrystal morphology. The effect of nanoparticles size (within the investigated range) is less pronounced, and mainly depends on ratio between inorganic core radius and thickness of organic shell. Figure 3 illustrates faceted mesocrystals prepared by the "gas phase diffusion technique" from a toluene dispersion of magnetite nanocrystals using oleic acid as surfactant and ethanol as non-solvent agent. The building blocks of the mesocrystals from all prepared nanocrystal batches were analyzed using Cs-corrected HRTEM and analytical ultracentrifugation (Table S1 and Figure 3, 4, S1S3). All nanoparticle batches show narrow size distributions, with nanocrystals from batch IV being significantly bigger on average than in other batches. While the truncated nanocubes from batches I-IV have uniform crystal faceting, they differ in their crystal habit with batch II showing the lowest degree of truncation and batches I and IV showing the highest. The shape of the particles from batches V and VI is best described as "semi-spherical" and were included in this study to examine the sole effect of interaction of oleic acid capped nanoparticles with solution molecules by excluding the effect of nanocrystal shape anisotropy and faceting.

Detailed results of TEM investigations of mesocrystalline films prepared from nanocrystals of all six batches by means of the solvent evaporation technique are shown in Figure S5. In case of 3D faceted mesocrystals (batches I-IV) prepared by the "gas phase diffusion technique", the morphology and the symmetry of the superlattice (i.e., translational order of nanoparticles) clearly changes when changing the crystal habit of the nanocrystals (Figure $\mathbf{3} \mathbf{~ b}, \mathbf{e}, \mathbf{h}, \mathbf{k}$ ). Starting with the smallest and most highly truncated nanocrystal batch, ${ }^{13}$ these building blocks lead to octahedral mesocrystals consistent with $f c c$ superlattice (Figure 3 a-c). The octahedral (111) face shows the $p 6 m m$ symmetry of the surface. Nanocrystal batch II presents the least truncated building blocks of the investigated nanocrystals, their size being slightly bigger than the nanocrystals of batch I (Figure S3).

Under the given conditions, these slightly truncated nanocrystals form mesocrystals with a morphology of tetragonal prisms (Figure 3 d-f, $b c t$ superlattice). The (001) basal face has a topology of a square lattice with the $p 4 \mathrm{~mm}$ symmetry. The truncation of nanocrystal batch III is in between nanocrystal batch I and II. These nanocrystals self-assemble to mesocrystals with a rhombohedral morphology (Figure $3 \mathbf{~ h}$ ). The symmetry of the mesocrystals basal rhombohedral face is $c 2 \mathrm{~mm}$ (Figure $\mathbf{3}$ i, FFT). Nanocrystal batch IV at the same time contains the biggest and highly truncated (slightly lower than batch I, Figure
S3) cubic building blocks (Figure $3 \mathbf{j}$ ). The morphology of the mesocrystals resulting from these nanocrystals can be described as tetragonal truncated pyramids. The SEM images of the surface layer of these self-assembled magnetite mesocrystals exhibit a $p 4 m m$ symmetry.

Remarkably, this data reveals that the morphology of mesocrystals and the related packing symmetry of the nanoparticles (e.g. translational order of nanoparticles within the mesocrystals) changes drastically with an increasing degree of truncation of the cubic building blocks. In case of self-assembly from toluene dispersion with increasing the degree of nanoparticles truncation the symmetry of superlattice changes from rhombohedral and $b c t$ and finally to $f c c$. The orientational order of the nanocrystals within the mesocrystalline arrays, however, is similar throughout nanocrystal batches I-IV (at least for mesocrystalline films), due to the same faceting of the nanocrystals (Figure S5).

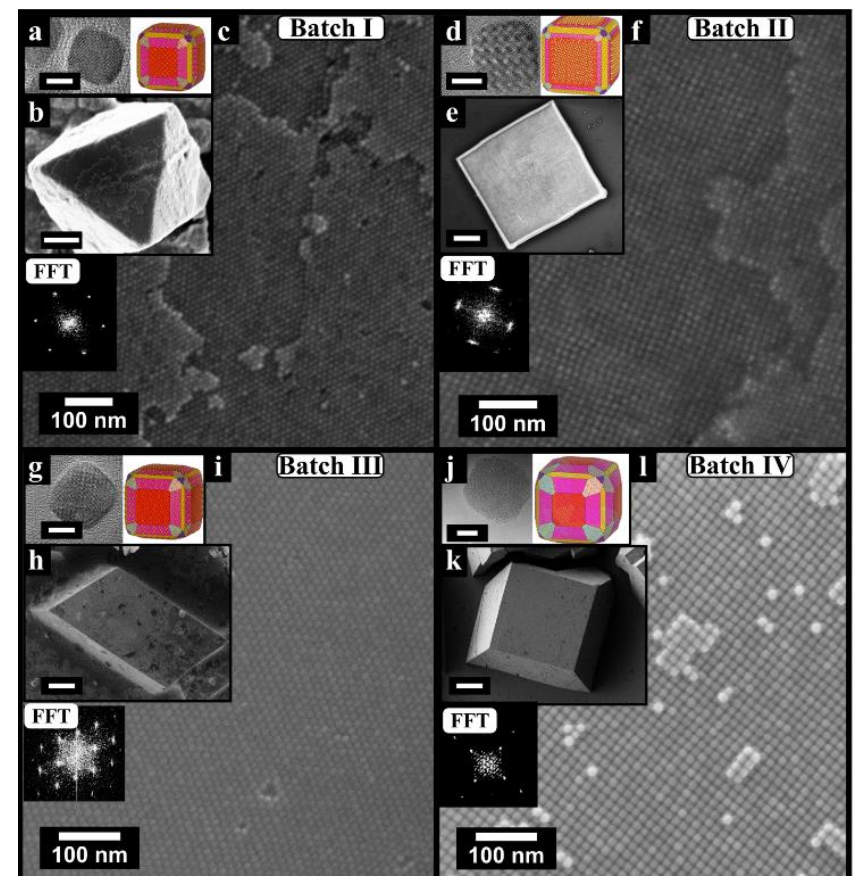

Figure 3. Illustration of mesocrystals crystallized from the toluene dispersion of different nanoparticle batches (I - IV). a), d), g) and j) Cs-corrected HRTEM images (insets: scale bars $=5 \mathrm{~nm}$ ) of the four nanoparticle batches together with models along [114]. b), e), h) and k) Mesocrystals crystallized under similar conditions in toluene and OA as surfactant. Different morphologies are visualized: b) an octahedral mesocrystal ( $f c c$ superlattice, insets: scale bar $=$ $500 \mathrm{~nm})$ ). e) a tetragonal prism (bct superlattice, inset: scale bar $=$ $5 \mu \mathrm{m})$ ). h) a rhombohedral mesocrystal (inset: scale bar $=10 \mu \mathrm{m}$ ) k) a truncated tetragonal pyramid (bct superlattice, inset: scale bar $=10 \mu \mathrm{m}) \mathbf{c})$, f), i) and l) HRSEM images and corresponding FFT of the projected mesocrystals faces showing the packing of the nanocubes. c) For mesocrystals of batch I, a $\{111\}$ face exhibing the p6mm plane symmetry, i) the rhombohedral mesocrystals exhibit on its (001) face a c2mm planar symmetry and f,) k) obtained from batch II and IV have a $\mathrm{p} 4 \mathrm{~mm}$ planar symmetry on its (001) basal face. 


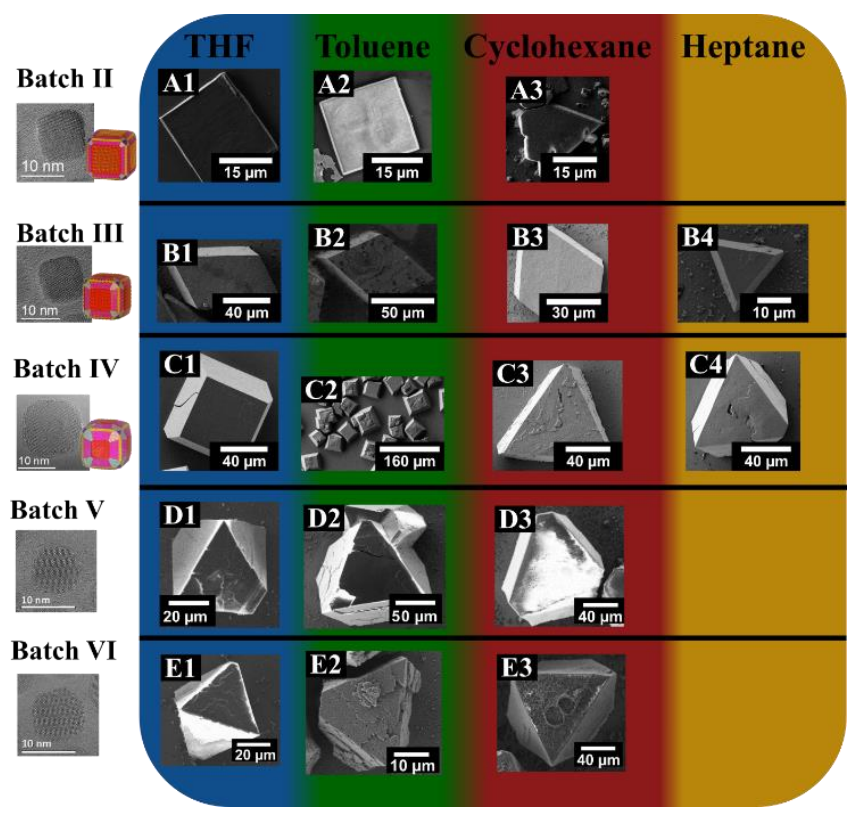

Figure 4. Mesocrystals prepared from nanocrystals dispersed in different solvents (batches II, III, IV, V, VI). Illustration of the changes of morphology of the mesocrystals by changing the solvent (from polar to nonpolar solvents). HRTEM images along [100] of the three nanocrystal batches and its approximated model (along [114]) are given (nanoparticles from batches V and VI show "semispherical" morphology). The size of the building blocks is presented in Table S1. A1 and A2 Batch II shows mesocrystals with a morphology of a tetragonal prisms when crystallized from toluene and THF. A3 The morphology changes to truncated trigonal pyramids when crystallized from cyclohexane. B1 and B2 Dispersed nanocrystals of batch III in THF and toluene lead to rhombohedral morphologies. B3 and B4 When crystallized from cyclohexane and heptane, the morphology changes to trigonal truncated pyramids. C1 and C2 Mesocrystals from nanocrystal batch IV show tetragonal truncated pyramids when crystallized from THF and toluene. C3 and C4 Trigonal truncated pyramids appear from nanocrystal batch IV when crystallized from cyclohexane and heptane. D1 - E3 Batches V and VI show mesocrystals with a morphology of a truncated trigonal pyramids and octahedra crystallized from THF, toluene and cyclohexane.

\section{Effect of dispersion agent on the morphology of 3D colloidal crystals.}

Further investigations of nanocrystal batches II-VI show that the morphology of colloidal crystals (namely mesocrystals for batches II-IV) and translational order of nanoparticles are also significantly affected by the nature of the dispersion agent. SEM and light microscope (LM) images nicely illustrate different morphologies of the aggregates (Figure 4 and Figure S6S8) crystallized from different media. The self-assembly of the nanoparticles from polar solvents such as THF and toluene (for our purposes solvent polarity is characterized by its dielectric constant and compared to oleic acid, Table S2) can lead to a variety of different morphologies, such as tetragonal prisms (batch II), rhombohedra (batch III), tetragonal truncated pyramids (batch IV) and trigonal truncated pyramids and octahedra (Figure 4 A1, A2, B1, B2, C1, C2, D1, D2, E1, E2).

The 2D symmetry of the (001) basal face of the mesocrystals from nanocrystal batches II and IV is $p 4 m m$, while the symmetry of the (001) face for the rhombohedral mesocrystal of nanocrystal batch III is $c 2 \mathrm{~mm}$ and for octahedral faces of batches V and VI is p6mm (Figure S6 d-i, S7 g-l, S8 g-l). In contrast, the morphology of the colloidal crystals is always a trigonal truncated pyramid (with $f c c$ symmetry of the superlattice), when the nanocrystals are aggregating from nonpolar dispersion agents such as cyclohexane and heptane (Figure 4 A3, B3, B4, C3, C4, D3, E3). All these mesocrystals show p6mm plane symmetry on the (111) basal face (Figure S6 a-c, $\mathbf{S 7}$ a-f and S8 a-f). Furthermore, the assembly process of the nanocrystals from nonpolar solvents is slower than for polar solvents. As an example, with THF as the dispersion agent, the complete self-assembly process occurred within four days, but it took eight days in case of toluene (keeping all other conditions the same, see the Experimental part). The complete crystallization time from cyclohexane and heptane solutions may even take up to a month or more. The difference of the morphology and the symmetry of the superlattice (e.g. structural polymorphism) cannot be exclusively explained by modified kinetics of the self-assembly process. Rather, we need to take into account the specific interaction of iron oxide nanocrystals stabilized by oleic acid molecules with surrounding media to explain the observed phenomena. ${ }^{29}$ Similar observations (of solvent impact onto mesocrystals shape and structure) were also reported for several other nanoparticle self-assemblies. ${ }^{37,53,71}$ Furthermore, molecular crystals can also be prepared with different morphologies and crystal structures (i.e. polymorph modifications) by crystallization from different solvents. ${ }^{68}$ It was suggested that the polarity and related dielectric constant of the solvents are crucial for this behavior of molecular and colloidal crystals. ${ }^{37} \mathrm{In}$ accordance to these reports and to our experimental observation, we suggest that the different morphologies of self-assembled magnetite mesocrystals obtained from different dispersion agents can be correlated to the polarity of dispersion agents and to the nature of the surfactant stabilizing the nanoparticle (i.e. the organic shell) (Table S2). Here, the interaction between the dispersion agent and the organic shell of the nanocrystals seems to be the most important parameter influencing the final morphology of the mesocrystals and the symmetry of the superlattice (while we used an excess of surfactant, which is also crucial for the successful formation of the mesocrystals ${ }^{13,37}$ ). Cyclohexane and heptane have a significantly lower dielectric constant than toluene and THF. In the case of mesocrystals from batches I-IV with morphologies of tetragonal prisms, rhombohedra, and tetragonal truncated pyramids, the dielectric constant of the solvents is similar or higher than that of oleic acid and the crystallization time is faster. While for the same batches, the mesocrystals with a shape of a trigonal truncated pyramid crystallize from the less polar solvents (e.g. cyclohexane and heptane). Based on this observation, we can also suggest that the effective shape of the nanoparticles is significantly affected by the interaction of stabilizing molecules (oleic acid) with the surrounding dispersion agent. It can be hypothesized that in nonpolar solvents the formed solvation shell changes the "effective shape" of the nanocrystals towards the spherical shape by smoothing the edges and vertices of the truncated nanocubes. This significantly affects the assembly process and the symmetry of the resulting superlattices. These observations are also consistent with the fact that, under the investigated conditions, the colloidal crystals formed from batches V and VI always have the $f c c$ structure and the morphology of trigonal truncated pyramids and/or octahedra independent from the dispersion agent. Furthermore, the kinetic of the crystallization process is also significantly affected. 

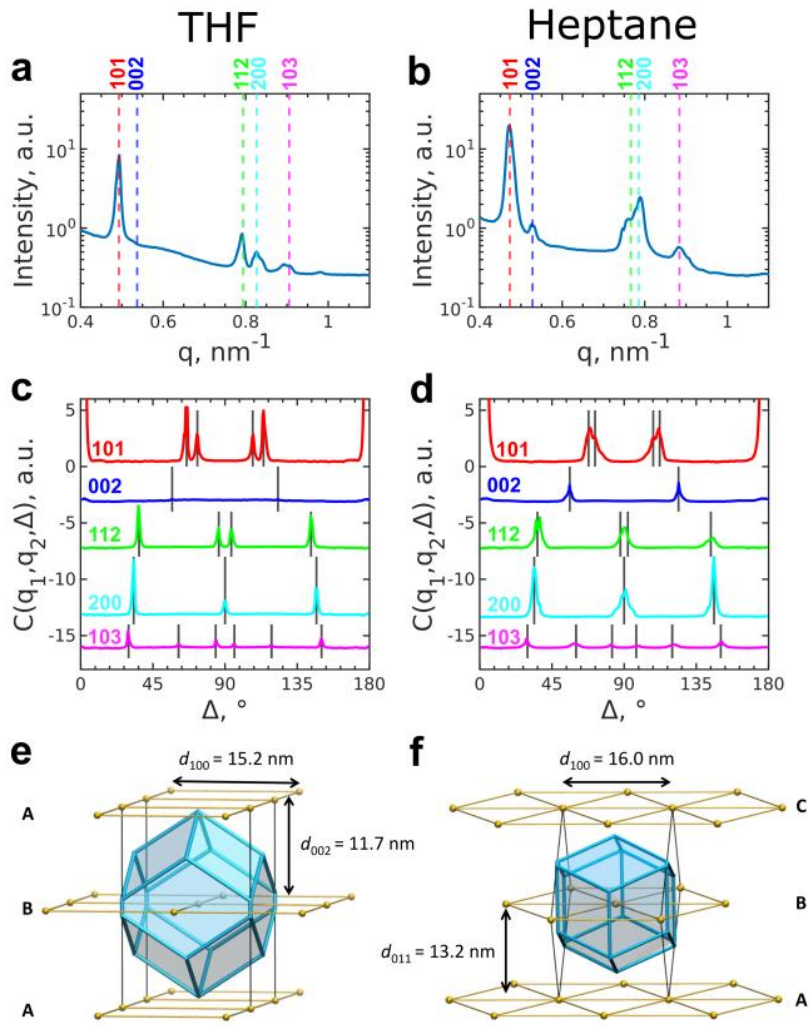

Figure 5. Superlattice structure revealed by AXCCA. a), b) Angular averaged radial profiles of the scattered intensity from the samples assembled from THF (a) and heptane (b). The dashed vertical lines show the peak positions for the structure with optimized unit cell parameters. c), d) Cross-correlation functions $C\left(q_{1}, q_{2}, \Delta\right)$ calculated for the intensities taken at the momentum transfer $q_{1}$ corresponding to the 101 Bragg peaks and $q_{2}$ corresponding to all peaks shown in panels a) and b), respectively. The graphs are offset for clarity. The black vertical lines show the peak position for the structure with optimized unit cell parameters. e), f) Structural models of mesocrystals grown from THF and heptane dispersion, illustrating the geometry of the Voronoi polyhedra and interparticle distance. The bct unit cells are shown in black lines.

\section{Effect of dispersion agent on the symmetry of superlattice and interparticle distance in mesocrystals.}

The mesocrystals grown from THF and heptane nanoparticles dispersions of batch IV were studied by XRD. Intensity distributions in 3D reciprocal space were collected from single grain samples (Figure S9) that allowed to determine the unit cell parameters not only from standard radial profile refinement, but also from more precise AXCCA (for details, see Methods and Supporting Information: S5 sub-chapter). Both samples could be refined to have $b c t$ superlattice (Figure 5). Unit cell parameters are $a=15.2 \pm 0.1 \mathrm{~nm}, c=23.4 \pm 0.3 \mathrm{~nm}$ for the sample grown from THF and $a=16.0 \pm 0.2 \mathrm{~nm}, c=23.5 \pm 0.6 \mathrm{~nm}$ for the sample grown from heptane (Figure $5 \mathrm{a}-\mathrm{d}$ ). The uncertainties refer to the determination of diffraction maxima, therefore more realistic error estimation could be gained from the broadness of peak profiles (Figure S12) and corresponds to approx. $0.4 \mathrm{~nm}$ and $1 \mathrm{~nm}$ (for $a$ and $c$ ) for THF sample, and 1.2 and $2 \mathrm{~nm}$ (for $a$ and $c$ ) for heptane one. Thus, the former sample has clearly tetragonal symmetry $(c / a=1.54)$, while the latter $(c / a=1.47)$ can be characterized as slightly distorted $f c c$ structure (cf. $\mathrm{c} / \mathrm{a}=\sqrt{2} \approx 1.41)$. The difference becomes even better visible from the metric in the unit cell parameter space (see Figure S13) and the geometry of the Voronoi polyhedra ${ }^{72}$ (Figure $\mathbf{5 e}, \mathbf{f})$. Interestingly, the comparison of lattice parameters of both mesocrystals, shows that parameter $a$ is higher for the heptane sample, while $c$ stays nearly the same. This might give an impression of "anisotropic" expansion, if one would consider the same growth scenario of both mesocrystals. However, the SEM images (Figure 3,4, S6-S8) clearly show, that the growth scenarios are indeed different. The layer-by-layer growth of mesocrystals takes place by stacking square nets $(\{001\}$ planes in $b c t$ structure) in THF (Figure 4, C1) and hexagonal nets ( $\{011\}$ planes in $b c t=\{111\}$ planes in $f c c$ structure) in heptane (Figure 4, C4). Thus, Figure 5 e, $\mathbf{f}$ shows that the heptane leads to isotropic increase of interparticle distances by ca. $0.8-1.5 \mathrm{~nm}$. Taking into account the size of cubic nanoparticles ("MinFeret" $=14.4 \mathrm{~nm}, \mathrm{SD}=0.7 \mathrm{~nm}$ ), the thickness of organic stabilizer between the particles extends from ca. $0.8 \mathrm{~nm}$ in THF to ca. 1.6 $\mathrm{nm}$ in heptane. Furthermore, XRD data indicate higher disorder in mesocrystals grown in heptane, by giving broader Bragg peaks in reciprocal space.

\section{Effect of dispersion agent on nanoparticle solvation shells.}

To investigate the complex interactions of nanoparticles with solvent molecules directly in liquid dispersion, we employed advanced double difference pair distribution function (dd-PDF) analysis of X-ray total scattering data and analytical ultracentrifugation (AUC). The dd-PDF analysis of nanoparticle dispersions allows to detect the restructuring of solvent molecules at the nanoparticle interface within the solvation shell. ${ }^{73}$ Due to broken symmetry and additional interactions with the nanoparticle surface, solvent molecules reorganize at interfaces. With increasing distance from the nanoparticles' surface, the bulk order is regained and the solvation shell signal vanishes. To study the solvation shells around iron oxide nanoparticles capped by oleic acid molecules, high energy X-ray scattering data was collected for nanocube dispersions, as well as the bulk dispersion media and dried nanopowders as backgrounds. For this dd-PDF analysis, we investigated batches V and VI in cyclohexane and in THF, with and without excess of oleic acid $(3 \mu \mathrm{L} / \mathrm{mL})$. The PDF is gained by Fourier Transformation of the total scattering data after background correction and normalization. In general, the PDF is a histogram of all interatomic distances within a sample. In the case of bulk solvents, both intramolecular distances and intermolecular distance correlations can be detected. The solvation shell signals in the dd-PDFs herein were extracted according to Thomä et al. ${ }^{74}$ Upon subtraction of the signal from the bulk solvent (pure THF or cyclohexane), all intramolecular distances of the solvent molecules get subtracted as the solvent molecules in the solvation shell do not dissociate or change compared to the bulk. However, their intermolecular arrangement changes, and thus, the medium-range molecular arrangement is affected, which becomes detectable as an electronic density oscillation in the PDF over ca. $30 \AA$ (Figure S15). For all samples, the extracted solvation shell signal contains broad oscillations. The collected signals for THF and cyclohexane were distinctly different. The size of the nanoparticles and the addition of extra oleic acid (in the concentration $3 \mu \mathrm{L} / \mathrm{mL}$ used for the self-assembly process) did not strongly affect the signal from the solvation shells (Figure S16). The derived solvation shell signals for THF and cyclohexane around nanoparticles from batch VI were exemplarily modelled with exponentially 
decaying sine waves according to Zobel et $a l .{ }^{73}$ and Thomä et $a l .{ }^{74}$ (Figure 6 a,b). At the distance, where the visible oscillation was decayed, a linear function was chosen for modelling (for more detailed information, see Figure S17). From the sinusoidal fit, we can readily obtain the extent of the restructuring, the overall number of restructured solvent layers (i.e. number of fitted oscillations) as well as the layer spacing (i.e. wavelength of the fitted oscillation). For the solvation shell formed around nanoparticles in THF, four restructured solvent layers were identified, while it is five layers in cyclohexane. Hence in cyclohexane, one layer more is affected than in THF. Further, layer spacings of $4.4 \AA$ and $5.0 \AA$ were obtained for THF and cyclohexane, respectively. These values are in good agreement with the linear sizes of THF and cyclohexane molecules estimated from van der Waals radii, which correlate well with electron density distributions, as known from literature (see insets Figure 6a, b). ${ }^{75}$ Moreover, it can be stated, that the extent of restructuring (solvation shell signal before decay) around the investigated iron oxide nanoparticles found with PDF analysis is ca. $8 \AA$ larger for cyclohexane than for THF. Thus, the solvation shell formed around the nanoparticles in cyclohexane could significantly modify the "effective shape" of the cubic nanoparticles towards sphere (Figure 6b).

In order to correlate these data with other physicochemical parameters of nanoparticles in different solvents including the effective size, density, and sedimentation behavior, AUC analysis was used. Figures S18 and S19 show the particle size distributions in different solvents as evaluated with two different evaluation algorithms. While $\mathrm{g}(\mathrm{s})$ yields a distribution, which is not corrected for the diffusion broadening of the sedimenting boundary, the 2 DSA-MC method corrects for this broadening so that individual components in the distribution can be seen and the distribution for each component is much narrower (Figures S18 and S19 upper row). In addition, 2 DSA-MC allows for plotting the distribution of the frictional coefficients $\mathrm{f} / \mathrm{f}_{0}$ versus the sedimentation coefficient distribution, while the PCSAMC method can calculate the partial specific volume of each species in the sedimentation coefficient distribution (Figures S18 and S19 lower row). It is obvious that several populations of nanoparticles exist for most of the samples but the differences between the most abundant species are small. The hydrodynamic diameter can then be calculated using the particle density (inverse $v_{b a r}$ ) using the formula $d_{H}=\sqrt{\frac{18 \eta s}{\rho_{p}-\rho_{s}}}$ (where $d_{H}$ is the hydrodynamic nanoparticle diameter, $s$ is sedimentation coefficient, $\eta$ the solvent viscosity, $\rho_{p}$ and $\rho_{s}$ are particle and solvent densities, respectively). These values are collected in Table S3. With the known diameter of the inorganic core of the nanoparticles obtained from TEM images, the thickness of the solvated organic shell can be calculated. It can be seen that significant differences exist for different solvents. In THF, the shell thickness is always the smallest, while it is largest for cyclohexane. There are differences between $\mathrm{g}(\mathrm{s})$ and 2 DSA-MC, but the general trend is independent of the AUC evaluation method. If compared to the persistence length of an oleic acid molecule (18.9 $\AA$ ), the oleic acid molecules could fold back on themselves because the interaction of the nonpolar chains with themselves is favored over the interaction with the solvent THF and have not an extended solvation shell (evidenced by the PDF analysis). Nevertheless, the molecules still provide steric stabilization. For the nonpolar solvent toluene and to a higher extent cyclohexane, the interaction of the nonpolar chains of oleic acid
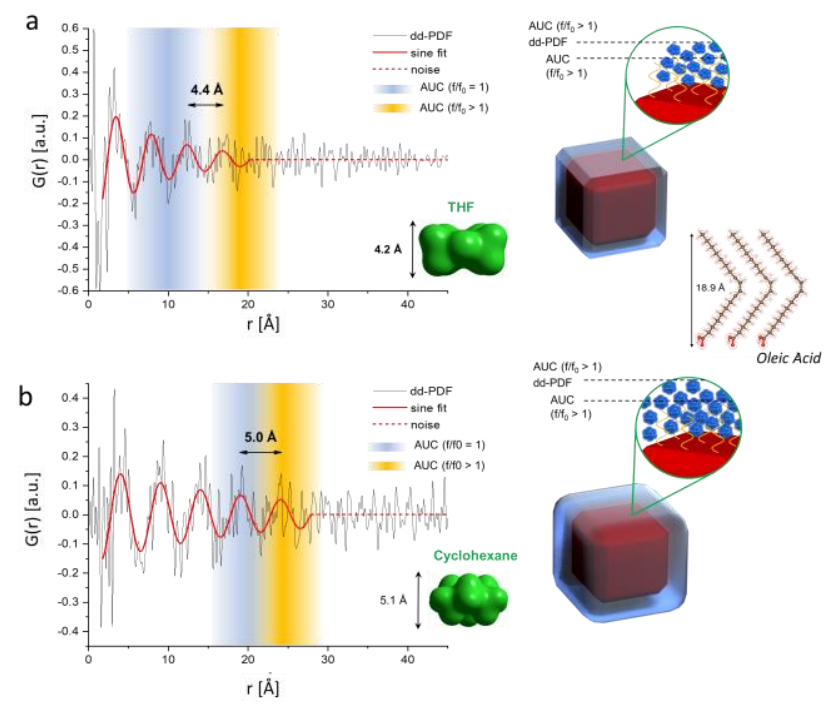

Figure 6. Comparison of solvation shell formed around iron oxide nanocrystals stabilized by oleic acid (Batch VI) in THF (a) and cyclohexane (b) solutions. (right insets) Schematic illustration of solvation shell around nanoparticles in different solvents and relations between the measured thickness values obtained by dd-PDF and AUC, and oleic acid molecules. Fitting of PDF curves shows that: for THF (a), the exponentially decaying sine wave describes the data from 1.8 to $20.2 \AA$ (solid line), while beyond a line function suffices (dashed line; no restructuring beyond $20.2 \AA$ ); for cyclohexane (b), the exponentially decaying sine wave extends out to $27.8 \AA$ (solid line). In the insets the side views of the simulated electron density distributions of THF (a) and respectively cyclohexane (b) are depicted in green colour. Evaluation of AUC data using 2DSA-MC analysis shows that: for THF, the calculated thickness of a "dense" shell (with $\mathrm{f} / \mathrm{f}_{0}=1.0$ ) corresponds to $10.5 \AA$, while the dynamic one (with $\mathrm{f} / \mathrm{f}_{0}>1.0$ ) is $19 \AA$ (close to the value obtained by dd-PDF); for cyclohexane (b), the calculated thickness of the "dense" shell (with $\mathrm{f} / \mathrm{f}_{0}=1.0$ ) corresponds to $22.5 \AA$, while the dynamic one (with $\mathrm{f} / \mathrm{f}_{0}>1.0$ ) is $24 \AA$ (close to the value obtained by dd-PDF).

molecules with the nonpolar solvent is favorable and the chains significantly extend into the solvent. This value varies a bit depending on the batch, but in general the stabilization shell extension in cyclohexane reaches from $17 \AA$ up to $23.5 \AA$ and is close to the values obtained by dd-PDF analysis. These values further agree well with the persistence length of a stretched oleic acid molecule in an extended solvation shell.

We can now obtain further information looking at the frictional ratios $f / f_{0}$ (Table S3). From TEM data, it is known that nanoparticles from both batches V and VI are spherical and therefore have a Perrin friction factor $\mathrm{P}$ of 1 . It is obvious that for particle populations with $f / f_{0}=1$ we can speak of a dense stabilization shell. But there are cases in each sample, where $f / f_{0}$ can be higher as 1, which indicates a significant amount of "mobile" solvent molecules associated with the particle shell. These are also the cases where a high thickness of the organic shell is observed compared to populations with $f / f_{0}=1$. We can, therefore, treat the solvation shell as dynamic in such a case where the oleic molecules extend into the solvent and also have a significant amount of solvent attached to them due to their favorable interaction. The extension of such a shell is in good agreement with the values obtained by PDF analysis (Figure 6a, b). Upon 
sedimentation of the particle, the solvent can flow through such shell causing increased friction and also, the oleic acid molecules have more degrees of freedom for movement due to their extension into the solution as those, which are folded back on themselves in the dense shell with $f / f_{0}=1$. With the exception of toluene for batch VI, the majority species in the distribution has $f / f_{0}>1$ for toluene and cyclohexane, while it is 1 for THF meaning that the shell is dynamic in toluene and cyclohexane, while it is dense in THF.

Based on these findings, we can finally verify the previously proposed hypothesis concerning the effect of solvent on the "effective shape" of the nanoparticles and changing the structuring and morphogenetic processes of colloidal crystals (incl. mesocrystals). Furthermore, our new findings could be essential for the further development of theories describing the aggregation behavior of nanoparticles in solution and potentially could explain the "non-additivity" of electrostatic, van der Waals and other interactions at the nanoscale (and deviations from classical DLVO theory ${ }^{29}$.

\section{CONCLUSIONS}

In summary, we successfully applied different crystallization methods to prepare 3D magnetite mesocrystals (type 1) and analyzed the crystallization conditions in detail. The nanocube building blocks were self-assembled either by solvent evaporation technique or gas phase diffusion technique and it was demonstrated that the crystallization process of mesocrystals is reversible. We have systematically investigated the effect of different nanoparticle and media parameters influencing the self-assembly process, morphology, and symmetry of the superlattice of crystallizing mesocrystals. It was shown that the faceting and habit of the nanocrystals is a crucial parameter affecting the symmetry of the superlattice and final morphology of the mesocrystals. However, the translational order of nanocrystals within mesocrystals may alter by changing of the dispersion agent (e.g., THF, toluene, cyclohexane, heptane). As an example, decreasing the solvent polarity, the shape of the mesocrystals assembled from slightly truncated cubic magnetite nanocrystals can change from tetragonal prisms and rhombohedra to trigonal truncated pyramids and octahedra, reflecting the changes of the superlattice symmetry (tetragonal, rhombohedral and cubic, respectively). Such changes of nanoparticle packing symmetry are to a certain extent in analogy to the structural polymorphism known for "classical" crystals. Furthermore, by using dd-PDF and AUC analysis of nanoparticle dispersions, it was shown that extension and density of the solvation shell of nanoparticles depend on the solvent polarity. For the nonpolar solvents like cyclohexane, the interaction of the nonpolar chains of oleic acid molecules with the solvent molecules is favourable and the chains significantly extend into the solvent. Furthermore, within the solvation shell around the nanoparticle, the extent of restructuring of solvent molecules is by ca. $8 \AA$ larger for cyclohexane than for THF, which significantly modifies the effective shape of the nanoparticles. Thus, in nonpolar solvents, the thick and "dynamic" solvation shell significantly affects the "effective shape" of the nanoparticles towards spheres and leads to the formation of colloidal crystals with a $f c c$ superlattice and the morphology of octahedra and/or truncated trigonal pyramids. This effect is responsible for the increase of the interparticle distances in mesocrystals grown from polar (e.g., THF) vs. non-polar solvents (e.g., heptane). These findings are consistent with our previously proposed phenomenological model, ${ }^{8,21}$ suggesting that the type of particle packing depends on their effective softness, which in turn (along with the nature of a solvent) has impact on the shape of the mesocrystals.

Finally, this fundamental research allows to understand basic principles of morphogenesis of self-assembled faceted mesocrystals built up from cubic magnetite nanoparticles with different degrees of truncation. These basic principles can be readily transferred to other nanocrystal systems. They will serve as a proxy system for the optimization of synthesis condition and synthesis of mesocrystals with defined structure and morphology.

\section{EXPERIMENTAL SECTION}

Synthesis of the nanocubes: The heating-up method was used for the preparation of the iron oxide nanocubes with different sizes and degrees of truncation according to literature. ${ }^{13,76,77}$

Synthesis of the mesocrystals, evaporation induced self-assembly: The evaporation induced self-assembly was performed according to literature. ${ }^{13}$ A nanocrystal dispersion $(5 \mathrm{mg} / \mathrm{mL})$ containing oleic acid $(1 \mu \mathrm{L} / \mathrm{mL})$ is dried slowly on top of a carbonfoiled TEM grid.

Synthesis of the mesocrystals, gas phase diffusion technique: In a glass vial containing a silicon snippet (orientation (100), CrysTec Kristalltechnologie), $400-500 \mu \mathrm{L}$ of the nanoparticle dispersion of a given nanoparticle and surfactant (oleic acid) concentration was injected. A typical experiment is performed with $5 \mathrm{mg} / \mathrm{mL}$ nanocrystal concentration and $3 \mu \mathrm{L} / \mathrm{mL}$ surfactant concentration. This glass vial was put into another glass vial containing the diffusion phase $(1.5-2.5 \mathrm{~mL})$. It was then stored until the nanoparticles were destabilized. Finally, the silicon snippet was removed and investigated.

Scanning Electron Microscopy: For the SEM images a Zeiss CrossBeam 1540XB was used reaching a resolution of up to 1.1 $\mathrm{nm}$. It is equipped with an InLens detector and SE2 detector.

Transmission Electron Microscopy: The TEM images were recorded using two different microscopes. The Zeiss Libra 120 can reach a point resolution of $0.34 \mathrm{~nm}$ with a $120 \mathrm{kV}$ Lanthanum hexaboride emitter and a Koehler illumination system. The high resolution TEM imaging of the nanoparticles was performed at $300 \mathrm{kV}$ acceleration voltage using a probe and image aberration corrected FEI TITAN ${ }^{3}$ transmission electron microscope. TEM images were analyzed with DigitalMicrograph ${ }^{\circledR}$ Gatan Microscopy Suite 3 software (Gatan Inc., ver. 3.41.2938.1). To calculate the core diameter of the nanocrystal batches TEM images were processed using Fiji software. The histograms were fitted using Gaussian function.

Single-crystal Small-angle X-ray Scattering: The SAXS measurements of the selected mesocrystals were performed by means of a Bruker AXS Nanostar diffractometer using $\mathrm{Cu} \mathrm{K} \alpha$ radiation. The analysis of SAXS pattern was performed using JEMS software. 
Single-crystal Wide-angle X-ray Diffraction: A single mesocrystal was mounted on a MicroLoop holder. The XRD images were collected by use of a Rigaku AFC7 diffractometer quipped with Mercury CCD Detector (Mo-K $\alpha$-radiation, $\lambda=0.71073 \AA$ ).

Analytical Ultracentrifugation: The AUC measurements were performed on an Optima XL-A (Beckman Coulter, Palo Alto, CA, United States) using absorbance optics at $7000 \mathrm{rpm}$ at $20{ }^{\circ} \mathrm{C} .12 \mathrm{~mm}$ double sector titanium centerpieces (Nanolytics, Potsdam, Germany) were used for all experiments. Sedfit (version $16.1 \mathrm{c}$ by Peter Schuck $^{78}$ ) was used for performing the $\mathrm{g}(\mathrm{s})$ distributions with the $1 \mathrm{~s}-\mathrm{g} *(\mathrm{~s})$ model and Tikhonov-Phillips regularization. ${ }^{79}$ UltraScan $3^{80}$ (Version 4.0, revision 2783) was used for performing the two-dimensional spectrum analysis (2DSA $)^{81}$, Custom Grid $(\mathrm{CG})^{82}$ and parametrically constrained spectrum analysis (PCSA). ${ }^{82}$ The 2DSA-, CG- and PCSAMonte Carlo (MC) analyses were performed with 50 iterations. The final fitted densities from PCSA-MC analyses were used for the calculations of the hydrodynamic diameter $\left(d_{H}\right)$ of nanoparticles. In another $d_{H}$ order, the real density of magnetite nanoparticles stabilized by oleic acid was estimated by taking into account that the oleic acid content was around $20 \mathrm{wt} \%$ (based on the results of chemical analysis). ${ }^{13}$

$X$-ray scattering experiment on the single grains. The X-ray experiment was performed on the cuboidal grains with all dimensions of about $1 \mu \mathrm{m}$ cut from the mesocrystals grown from THF and heptane dispersions by focused ion beam (see Supporting Information: S4 sub-chapter for details). The scattered intensity in full 3D reciprocal space of the colloidal crystal grains was measured the same way as described in Ref. ${ }^{45}$ The experiment was performed at P10 Coherence Applications beamline at PETRA III storage ring (DESY, Germany). Monochromatic Xrays of $10.235 \mathrm{keV}$ were focused down to $\sim 2.5 \times 2.0 \mu \mathrm{m}^{2}$ at the sample position completely covering slightly smaller colloidal crystal grain. The colloidal crystal grain was fixed on a tungsten tip mounted on a rotation stage and rotated around the vertical axis. At each angular position, the $2 \mathrm{D}$ far-field diffraction patterns were recorded by the EIGER X 4M detector positioned $4.95 \mathrm{~m}$ downstream from the sample. The sample was rotated by steps of $0.33^{\circ}$ over a range of $180^{\circ}$ and, by that, the full 3D diffraction pattern was measured. At each angular position, a series of 3 frames of $1 \mathrm{~s}$ exposure each were measured, corresponding to $3 \mathrm{~s}$ accumulated exposure to the non-attenuated $\mathrm{X}$ ray beam. The sample was cooled using a liquid nitrogen cryostat, in order to avoid radiation damage of the organic ligands (stabilizing nanocrystals) which could induce the coalescence of nanoparticles and destroy the superlattice ordering.

Angular X-ray Cross-Correlation Analysis. AXCCA was performed as described in Ref. ${ }^{45}$ Cross-correlation functions were calculated between the intensities taken at the brightest first peak momentum transfer $\left(q \approx 0.5 \mathrm{~nm}^{-1}\right)$ and at other momentum transfers in the range of $q=0.4-1.3 \mathrm{~nm}^{-1}$ with the step of $0.005 \mathrm{~nm}^{-1}$. The resulting cross-correlation map was represented in $(q, \Delta)$-coordinates. The expected peak positions for the selected superlattice model in these coordinates were calculated and the cross-correlation intensities in these positions were calculated by interpolation of the experimental map. The optimal unit cell parameters of the superlattice were found by maximization of the total intensities at the expected peak positions (see Supporting Information for details).
PDF data acquisition and processing. XRD measurements of dispersions of iron oxide nanocubes and pure solvents were carried out at $68 \mathrm{keV}(0.1823 \AA)$ at the European Synchrotron Radiation Facility (ESRF) at beamline ID15-A using a PILATUS3 $\mathrm{X}$ CdTe $2 \mathrm{M}$ detector $\left(253.7 \times 288.8 \mathrm{~mm}^{2}\right.$ sensitive area, $172 \times 172 \mu \mathrm{m}^{2}$ pixel size). XRD data of a corresponding dry iron oxide nanopowder was acquired at beamline I15-1 (XPDF) at $65.4 \mathrm{keV}(0.18957 \AA)$ at Diamond Light Source equipped with a Perkin Elmer detector XRD 4343 CT $\left(432 \times 432 \mathrm{~mm}^{2}\right.$ active area, $150 \times 150 \mu \mathrm{m}^{2}$ pixel size). Each data set at ESRF was collected for a total of 3 minutes and at Diamond Light Source for 6 minutes. Thereby, 20 data collections of 9 seconds (ESRF) and 12 data collections of 30 seconds (Diamond) each were performed and then averaged. All samples were measured in $1 \mathrm{~mm}$ Kapton ${ }^{\circledR}$ capillaries. NIST chromium(III) oxide standards (ESRF) and NIST silicon standards (Diamond) were used for distance calibration and instrumental resolution determination. For data processing of ESRF data the following software packages were used: for masking Fit2D; for calibration pyFAIcalib2; and for radial integration xpdtools. For data from Diamond Light Source calibration, radial integration, masking and normalization of the data was done in the DAWN software package. ${ }^{83}$ PDF processing was carried out with $x$ PDFsuite ${ }^{84}$ and fitting in IgorPro by WaveMetrics. All data was treated in the same way during data analysis. The powder PDF of the iron oxide nanopowder was scaled to the experimental d-PDFs each with an individual scale factor $\mathrm{y}_{1}$ in the distance range $>20 \AA$. The iron oxide nanopowders data was collected on nanoparticles of batch IV, while the dispersions measured contained nanoparticles of batches V and VI, as no powder of the latter cubes was available. The difference between the PDFs of 8-100 and $150 \AA$ cubes, in particular over the interesting distance range of $30 \AA$ is negligible as the shape functions of the cubes are pretty similar in this range. Moreover, the shape functions are constantly decaying over $r$, while the solvent restructuring features a sinusoidal structural fingerprint. Therefore, for means of this data analysis, where the PDFs are subtracted in real space, any errors which are of importance to the interpretations drawn due to the subtraction of the PDF of a slightly offset iron oxide powder size, can be ruled out. According to Thomä et al. ${ }^{74}$ the ddPDFs of the different dispersions can be compared by mutual scaling of the powder PDFs contribution to the overall PDF. For this, we again use the scale factor $\mathrm{y}_{2}$ and in this case the intensity of the IONP PDF peak at $28.52 \AA$. All samples were scaled to an intensity of 0.3 at this point, since this value was the average value for all samples. The description of the solvation layers is based on an exponentially decaying sine wave according to Thomä et $a l .{ }^{74}$ The high frequency ripples visible in the ddPDFs is high-frequency noise due to the low concentration of the dispersions and does not contain any structural signal.

\section{ASSOCIATED CONTENT}

The Supporting Information is available free of charge

Additional data including SEM and TEM images, dd-PDF and AUC evaluation, and details of AXCCA

\section{AUTHOR INFORMATION}

\section{Corresponding Author}

* Elena V. Sturm

e-mail: elena.sturm@uni-konstanz.de 


\section{Author Contributions}

The manuscript was written through contributions of all authors. All authors have given approval to the final version of the manuscript.

\section{ACKNOWLEDGMENT}

We hereby acknowledge the DFG (Deutsche Forschungsgemeinschaft) for financial support (SFB1214 Project B1). We thank Clara Schlotheuber for editorial help and fruitful discussions. We also thank Dr. Danny Haubold and Ramon Zimmermanns for being a great help in laboratory work. SS and AL acknowledge funding from the European Research Council (ERC) under the Horizon 2020 research and innovation program of the European Union (grant agreement number 715620). M. Z. acknowledges support by the Bavarian Academy for Sciences and Humanities. M.Z. and S. T. acknowledge beamtime at ID15-ERH at European Synchrotron Radiation Facility and I15-1 at Diamond Lightsource, as well as support from the respective beamline scientists Gavin Vaughan and Phil Chater. We acknowledge DESY (Hamburg, Germany), a member of the Helmholtz Association HGF, for the provision of experimental facilities. Parts of this research were carried out at PETRA III synchrotron facility and we would like to thank the beamline staff and especially Michael Sprung for assistance in using Coherence Application P10 beamline. This work was supported by the Helmholtz Associations Initiative Networking Fund (grant No. HRSF-0002) and the Russian Science Foundation (grant No. 18-41-06001). E.V.S. thanks the Zukunftskolleg at the University of Konstanz and "Konstanzia Transition" programme of equal opportunity council for financial support. Last but not least, we thank the Bio Imaging Center Constance and Stefan Helfrich to help us to evaluate light microscope images, quantitatively.

\section{REFERENCES}

1. Talapin, D. V., Nanocrystal solids: A modular approach to materials design. MRS Bull. 2012, 37 (1), 63-71.

2. Talapin, D. V., Lee, J.S., Kovalenko, M., Shevchenko, E., Prospects of Colloidal Nanocrystals for Electronic and Optoelectronic

Applications. Chem. Rev. 2010, 110, 389-458.

3. Reichhelm, A.; Haubold, D.; Eychmüller, A., Ligand Versatility in Supercrystal Formation. Adv. Funct. Mater. 2017, 27 (39), 1700361.

4. Polarz, S., Shape Matters: Anisotropy of the Morphology of Inorganic Colloidal Particles - Synthesis and Function. Adv. Funct. Mater. 2011, 21 (17), 3214-3230.

5. Talapin, D. V.; Shevchenko, E. V., Introduction: Nanoparticle Chemistry. Chem. Rev. 2016, 116 (18), 10343-10345. 6. Trepka, B.; Erler, P.; Selzer, S.; Kollek, T.; Boldt, K.; Fonin, M.; Nowak, U.; Wolf, D.; Lubk, A.; Polarz, S., Nanomorphology Effects in Semiconductors with Native Ferromagnetism: Hierarchical Europium (II) Oxide Tubes Prepared via a Topotactic Nanostructure Transition. Adv. Mater. 2018, 30 (1).

7. Kagan, C. R.; Lifshitz, E.; Sargent, E. H.; Talapin, D. V., Building devices from colloidal quantum dots. Science 2016, 353 (6302).

8. Simon, P.; Rosseeva, E.; Baburin, I. A.; Liebscher, L.; Hickey, S. G.; Cardoso-Gil, R.; Eychmüller, A.; Kniep, R.; Carrillo-Cabrera, W., PbS-organic mesocrystals: the relationship between nanocrystal orientation and superlattice array. Angew. Chem. Int. Ed. 2012, 51 (43), 10776-81.

9. Boles, M. A.; Ling, D.; Hyeon, T.; Talapin, D. V., The surface science of nanocrystals. Nat Mater 2016, 15 (2), 141-53.

10. Dreyer, A.; Feld, A.; Kornowski, A.; Yilmaz, E. D.; Noei, H.; Meyer, A.; Krekeler, T.; Jiao, C.; Stierle, A.; Abetz, V.; Weller, H.; Schneider, G. A., Organically linked iron oxide nanoparticle supercrystals with exceptional isotropic mechanical properties. Nat. Mater. 2016, 15 (5), 522-528.

11. Abécassis, B., Three-Dimensional Self Assembly of Semiconducting Colloidal Nanocrystals: From Fundamental Forces to Collective Optical Properties. ChemPhysChem 2016, 17 (5), 618-631.

12. Boles, M. A.; Engel, M.; Talapin, D. V., Self-Assembly of Colloidal Nanocrystals: From Intricate Structures to Functional Materials. Chem. Rev. 2016, 116 (18), 11220-11289.

13. Brunner, J.; Baburin, I. A.; Sturm, S.; Kvashnina, K.; Rossberg, A.; Pietsch, T.; Andreev, S.; Sturm née Rosseeva, E.; Cölfen, H., Self-Assembled Magnetite Mesocrystalline Films: Toward Structural Evolution from 2D to 3D Superlattices. Adv. Mater. Interfaces 2017, 4 (1), 1600431.

14. Quan, Z. W.; Fang, J. Y., Superlattices with nonspherical building blocks. Nano Today 2010, 5 (5), 390-411.

15. Imai, H.; Tochimoto, N.; Nishino, Y.; Takezawa, Y.; Oaki, Y., Oriented Nanocrystal Mosaic in Monodispersed CaCO3 Microspheres with Functional Organic Molecules. Cryst. Growth Des. 2012, 12 (2), 876-882.

16. Haubold, D.; Reichhelm, A.; Weiz, A.; Borchardt, L.; Ziegler, C.; Bahrig, L.; Kaskel, S.; Ruck, M.; Eychmüller, A., The Formation and Morphology of Nanoparticle Supracrystals. Adv. Funct. Mater. 2016, 26 (27), 4890-4895.

17. Bergström, L.; Sturm nee Rosseeva, E. V.; SalazarAlvarez, G.; Cölfen, H., Mesocrystals in Biominerals and Colloidal Arrays. Acc. Chem. Res. 2015, 48 (5), 1391-402.

18. Zhang, J.; Zhu, J.; Li, R.; Fang, J.; Wang, Z., EntropyDriven Pt3Co Nanocube Assembles and Thermally Mediated Electrical Conductivity with Anisotropic Variation of the Rhombohedral Superlattice. Nano Lett. 2017, 17 (1), 362-367.

19. Cölfen, H.; Antonietti, M., Mesocrystals and Nonclassical Crystallization. John Wiley \& Sons: Chichester, 2008.

20. De Yoreo, J. J.; Gilbert, P. U. P. A.; Sommerdijk, N. A. J. M.; Penn, R. L.; Whitelam, S.; Joester, D.; Zhang, H.; Rimer, J. D.; Navrotsky, A.; Banfield, J. F.; Wallace, A. F.; Michel, F. M.; Meldrum, F. C.; Cölfen, H.; Dove, P. M., Crystallization by particle attachment in synthetic, biogenic, and geologic environments. Science 2015, 349 (6247), aaa6760.

21. Sturm, E. V.; Cölfen, H., Mesocrystals: structural and morphogenetic aspects. Chem. Soc. Rev. 2016, 45 (21), 5821-5833. 22. Cölfen, H.; Antonietti, M., Mesocrystals: Inorganic superstructures made by highly parallel crystallization and controlled alignment. Angew. Chem. Int. Ed. 2005, 44 (35), 55765591.

23. Sturm , E.; Cölfen, H., Mesocrystals: Past, Presence, Future. Crystals 2017, 7 (7), 207.

24. Gantapara, A. P.; de Graaf, J.; van Roij, R.; Dijkstra, M., Phase behavior of a family of truncated hard cubes. J. Chem. Phys. 2015, 142 (5), 16.

25. Coquerel, G., Crystallization of molecular systems from solution: phase diagrams, supersaturation and other basic concepts. Chem. Soc. Rev. 2014, 43 (7), 2286-2300.

26. Wang, T.; LaMontagne, D.; Lynch, J.; Zhuang, J.; Cao, Y. C., Colloidal superparticles from nanoparticle assembly. Chem. Soc. Rev. 2013, 42 (7), 2804-2823.

27. Levine, S.; Dube, G. P., Interaction between two hydrophobic colloidal particles, using the approximate Debye- 
Huckel theory. I. General properties. Trans. Faraday Soc. 1939, 35 (0), 1125-1140.

28. Israelachvili, J. N.; Mitchell, D. J.; Ninham, B. W., Theory of self-assembly of hydrocarbon amphiphiles into micelles and bilayers. J. Chem. Soc., Faraday Trans. 2 1976, 72, 1525-1568. 29. Batista, C. A. S.; Larson, R. G.; Kotov, N. A., Nonadditivity of nanoparticle interactions. Science 2015, 350 (6257), 11.

30. Disch, S.; Wetterskog, E.; Hermann, R. P.; SalazarAlvarez, G.; Busch, P.; Bruckel, T.; Bergström, L.; Kamali, S., Shape induced symmetry in self-assembled mesocrystals of iron oxide nanocubes. Nano Lett. 2011, 11 (4), 1651-6.

31. Li, R.; Bian, K.; Wang, Y.; Xu, H.; Hollingsworth, J. A.; Hanrath, T.; Fang, J.; Wang, Z., An Obtuse Rhombohedral Superlattice Assembled by Pt Nanocubes. Nano Lett. 2015, 15 (9), 6254-60.

32. Wei, J.; Schaeffer, N.; Pileni, M.-P., Solvent-Mediated Crystallization of Nanocrystal 3D Assemblies of Silver Nanocrystals: Unexpected Superlattice Ripening. Chem. Mater. 2015, 28 (1), 293-302.

33. Bian, K.; Li, R.; Fan, H., Controlled Self-Assembly and Tuning of Large PbS Nanoparticle Supercrystals. Chem. Mater. 2018, 30 (19), 6788-6793.

34. Hanske, C.; Hill, E. H.; Vila-Liarte, D.; GonzálezRubio, G.; Matricardi, C.; Mihi, A.; Liz-Marzán, L. M., SolventAssisted Self-Assembly of Gold Nanorods into Hierarchically Organized Plasmonic Mesostructures. ACS Appl. Mater. Interfaces 2019, 11 (12), 11763-11771.

35. Huang, X.; Wang, Z., Supercrystallography-Based Decoding of Structure and Driving Force of Nanocrystal Assembly. Materials 2019, 12 (22), 3771-3771.

36. Imai, H.; Matsumoto, R.; Takasaki, M.; Tsukiyama, K.; Sawano, K.; Nakagawa, Y., Evaporation-driven manipulation of nanoscale brickwork structures for the design of $1 \mathrm{D}, 2 \mathrm{D}$, and 3D microarrays of rectangular building blocks. CrystEngComm 2019, 21 (45), 6905-6914.

37. Lv, Z.-P.; Kapuscinski, M.; Bergström, L., Tunable assembly of truncated nanocubes by evaporation-driven poorsolvent enrichment. Nat. Commun. 2019, 10 (1), 4228-4228.

38. Zhu, H.; Fan, Z.; Yu, L.; Wilson, M. A.; Nagaoka, Y.; Eggert, D.; Cao, C.; Liu, Y.; Wei, Z.; Wang, X.; He, J.; Zhao, J.; Li, R.; Wang, Z.; Grünwald, M.; Chen, O., Controlling Nanoparticle Orientations in the Self-Assembly of Patchy Quantum Dot-Gold Heterostructural Nanocrystals. J. Am. Chem. Soc. 2019, 141 (14), 6013-6021.

39. Andre, A.; Zherebetskyy, D.; Hanifi, D.; He, B.; Khoshkhoo, M. S.; Jankowski, M.; Chasse, T.; Wang, L. W.; Schreiber, F.; Salleo, A.; Liu, Y.; Scheele, M., Toward Conductive Mesocrystalline Assemblies: PbS Nanocrystals Cross-Linked with Tetrathiafulvalene Dicarboxylate. Chem. Mater. 2015, 27 (23), 8105-8115.

40. Tebbe, M.; Lentz, S.; Guerrini, L.; Fery, A.; AlvarezPuebla, R. A.; Pazos-Perez, N., Fabrication and optical enhancing properties of discrete supercrystals. Nanoscale 2016, 8 (25), 1270212709.

41. Mukharamova, N.; Lapkin, D.; Zaluzhnyy, I. A.; André, A.; Lazarev, S.; Kim, Y. Y.; Sprung, M.; Kurta, R. P.; Schreiber, F.; Vartanyants, I. A.; Scheele, M., Revealing Grain Boundaries and Defect Formation in Nanocrystal Superlattices by Nanodiffraction. Small 2019, 15 (50), 1904954.

42. Zaluzhnyy, I. A.; Kurta, R. P.; André, A.; Gorobtsov, O. Y.; Rose, M.; Skopintsev, P.; Besedin, I.; Zozulya, A. V.; Sprung, M.; Schreiber, F.; Vartanyants, I. A.; Scheele, M., Quantifying Angular Correlations between the Atomic Lattice and the Superlattice of Nanocrystals Assembled with Directional Linking. Nano Lett. 2017, 17 (6), 3511-3517.
43. Zaluzhnyy, I. A.; Kurta, R. P.; Scheele, M.; Schreiber, F.; Ostrovskii, B. I.; Vartanyants, I. A., Angular X-ray CrossCorrelation Analysis (AXCCA): Basic Concepts and Recent Applications to Soft Matter and Nanomaterials. Materials 2019, 12 (21), 3464.

44. Kurta, R. P.; Altarelli, M.; Vartanyants, I. A., Structural analysis by $\mathrm{x}$-ray intensity angular cross correlations. In $A d v$ Chem. Phys., Rice, S. A.; Dinner, A. R., Eds. John Wiley \& Sons, Inc., Hoboken: New Jersey, 2016; Vol. 161, pp 1-39.

45. Carnis, J.; Kirner, F.; Lapkin, D.; Sturm, S.; Kim, Y. Y.; Baburin, I. A.; Khubbutdinov, R.; Ignatenko, A.; Iashina, E.; Mistonov, A.; Steegemans, T.; Wieck, T.; Gemming, T.; Lubk, A.; Lazarev, S.; Sprung, M.; Vartanyants, I. A.; Sturm, E. V., Exploring the 3D structure and defects of a self-assembled gold mesocrystal by coherent $\mathrm{X}$-ray diffraction imaging. Nanoscale 2021.

46. de Nijs, B.; Dussi, S.; Smallenburg, F.; Meeldijk, J. D.; Groenendijk, D. J.; Filion, L.; Imhof, A.; van Blaaderen, A.; Dijkstra, M., Entropy-driven formation of large icosahedral colloidal clusters by spherical confinement. Nat. Mater. 2015, 14 (1), 56-60.

47. Agthe, M.; Plivelic, T. S.; Labrador, A.; Bergström, L.; Salazar-Alvarez, G., Following in Real Time the Two-Step Assembly of Nanoparticles into Mesocrystals in Levitating Drops. Nano Lett. 2016, 16 (11), 6838-6843.

48. Agthe, M.; Hoydalsvik, K.; Mayence, A.; Karvinen, P.; Liebi, M.; Bergström, L.; Nygard, K., Controlling Orientational and Translational Order of Iron Oxide Nanocubes by Assembly in Nanofluidic Containers. Langmuir 2015, 31 (45), 12537-43.

49. Guillaussier, A.; Yu, Y.; Voggu, V. R.; Aigner, W.; Cabezas, C. S.; Houck, D. W.; Shah, T.; Smilgies, D.-M.; Pereira, R. N.; Stutzmann, M.; Korgel, B. A., Silicon Nanocrystal Superlattice Nucleation and Growth. Langmuir 2017, 33 (45), 13068-13076.

50. Zanaga, D.; Bleichrodt, F.; Altantzis, T.; Winckelmans, N.; Palenstijn, W. J.; Sijbers, J.; de Nijs, B.; van Huis, M. A.; Sánchez-Iglesias, A.; Liz-Marzán, L. M.; van Blaaderen, A.; Joost Batenburg, K.; Bals, S.; Van Tendeloo, G., Quantitative 3D analysis of huge nanoparticle assemblies. Nanoscale 2016, 8 (1), 292-299.

51. Born, P.; Munoz, A.; Cavelius, C.; Kraus, T., Crystallization Mechanisms in Convective Particle Assembly. Langmuir 2012, 28 (22), 8300-8308.

52. Choi, J. J.; Bealing, C. R.; Bian, K.; Hughes, K. J.; Zhang, W.; Smilgies, D. M.; Hennig, R. G.; Engstrom, J. R.; Hanrath, T., Controlling nanocrystal superlattice symmetry and shape-anisotropic interactions through variable ligand surface coverage. J. Am. Chem. Soc. 2011, 133 (9), 3131-8.

53. Quan, Z.; Xu, H.; Wang, C.; Wen, X.; Wang, Y.; Zhu, J.; Li, R.; Sheehan, C. J.; Wang, Z.; Smilgies, D. M.; Luo, Z.; Fang, J., Solvent-mediated self-assembly of nanocube superlattices. J. Am. Chem. Soc. 2014, 136 (4), 1352-9.

54. Wetterskog, E.; Klapper, A.; Disch, S.; Josten, E.; Hermann, R. P.; Rücker, U.; Brückel, T.; Bergström, L.; SalazarAlvarez, G., Tuning the structure and habit of iron oxide mesocrystals. Nanoscale 2016, 8 (34), 15571-15580.

55. Lee, B.; Littrell, K.; Sha, Y.; Shevchenko, E. V., Revealing the Effects of the Non-solvent on the Ligand Shell of Nanoparticles and Their Crystallization. J. Am. Chem. Soc. 2019, 141 (42), 16651-16662.

56. Huang, X.; Zhu, J.; Ge, B.; Deng, K.; Wu, X.; Xiao, T.; Jiang, T.; Quan, Z.; Cao, Y. C.; Wang, Z., Understanding Fe3O4 Nanocube Assembly with Reconstruction of a Consistent Superlattice Phase Diagram. J. Am. Chem. Soc. 2019, 141 (7), 3198-3206. 
57. Huang, X.; Wang, Z., Supercrystallography-Based Decoding of Structure and Driving Force of Nanocrystal Assembly. Materials 2019, 12 (22), 3771.

58. Josten, E.; Wetterskog, E.; Glavic, A.; Boesecke, P.; Feoktystov, A.; Brauweiler-Reuters, E.; Rücker, U.; SalazarAlvarez, G.; Brückel, T.; Bergström, L., Superlattice growth and rearrangement during evaporation-induced nanoparticle selfassembly. Sci. Rep. 2017, 7 (1), 2802.

59. Weidman, M. C.; Smilgies, D.-M.; Tisdale, W. A., Kinetics of the self-assembly of nanocrystal superlattices measured by real-time in situ X-ray scattering. Nat. Mater. 2016, 15 (7), 775781.

60. Agthe, M.; Wetterskog, E.; Bergström, L., Following the Assembly of Iron Oxide Nanocubes by Video Microscopy and Quartz Crystal Microbalance with Dissipation Monitoring. Langmuir 2017, 33 (1), 303-310.

61. Goubet, N.; Richardi, J.; Albouy, P.-A.; Pileni, M.-P., How to predict the growth mechanism of supracrystals from gold nanocrystals. J. Phys. Chem. Lett. 2011, 2 (5), 417-422.

62. Agarwal, U.; Escobedo, F. A., Mesophase behaviour of polyhedral particles. Nat. Mater. 2011, 10 (3), 230-235.

63. Faure, B.; Salazar-Alvarez, G.; Bergström, L., Hamaker Constants of Iron Oxide Nanoparticles. Langmuir 2011, 27 (14), $8659-8664$

64. Brunner, J. J.; Krumova, M.; Cölfen, H.; Sturm, E. V., Magnetic field-assisted assembly of iron oxide mesocrystals: a matter of nanoparticle shape and magnetic anisotropy. Beilstein J. Nanotechnol. 2019, 10, 894-900.

65. Opel, J.; Brunner, J.; Zimmermanns, R.; Steegmans, T.; Sturm, E.; Kellermeier, M.; Cölfen, H.; García - Ruiz, J. M., Symbiosis of Silica Biomorphs and Magnetite Mesocrystals. Adv. Funct. Mater. 2019, 29 (37), 1902047-1902047.

66. Sturm, S.; Siglreitmeier, M.; Wolf, D.; Vogel, K.; Gratz, M.; Faivre, D.; Lubk, A.; Büchner, B.; Sturm, E. V.; Cölfen, H., Magnetic Nanoparticle Chains in Gelatin Ferrogels: Bioinspiration from Magnetotactic Bacteria. Adv. Funct. Mater. 2019, 29 (45).

67. Kitaigorodkij, A. I., Organic Chemical Crystallography. Consultants Bureau 1961; p 541.

68. Spingler, B.; Schnidrig, S.; Todorova, T.; Wild, F., Some thoughts about the single crystal growth of small molecules. CrystEngComm 2012, 14 (3), 751-757.

69. Brunner, J.; Maier, B.; Rosenberg, R.; Sturm, S.; Cölfen, H.; Sturm, E. V., Nonclassical Recrystallization. Chem. Eur. 2020, 26 (66), 15242-15248.

70. Förster, S.; Timmann, A.; Schellbach, C.; Frömsdorf, A.; Kornowski, A.; Weller, H.; Roth, S. V.; Lindner, P., Order causes secondary Bragg peaks in soft materials. Nat. Mater. 2007, $6(11), 888-893$.

71. Chan, H.; Demortiere, A.; Vukovic, L.; Kral, P.; Petit, C., Colloidal nanocube supercrystals stabilized by multipolar Coulombic coupling. ACS Nano 2012, 6 (5), 4203-13.
72. Blatov, V. A., Voronoi-dirichlet polyhedra in crystal chemistry: theory and applications. Crystallography Reviews 2007, 10 (4), 249-318.

73. Zobel, M.; Neder, R. B.; Kimber, S. A., Universal solvent restructuring induced by colloidal nanoparticles. Science 2015, 347 (6219), 292-4.

74. Thomä, S. L. J.; Krauss, S. W.; Eckardt, M.; Chater, P.; Zobel, M., Atomic insight into hydration shells around facetted nanoparticles. Nat. Commun. 2019, 10 (1), 995.

75. Tkatchenko, A.; Scheffler, M., Accurate molecular van der Waals interactions from ground-state electron density and freeatom reference data. Phys. Rev. Lett. 2009, 102 (7), 073005.

76. Park, Y. I.; Piao, Y.; Lee, N.; Yoo, B.; Kim, B. H.; Choi, S. H.; Hyeon, T., Transformation of hydrophobic iron oxide nanoparticles to hydrophilic and biocompatible maghemite nanocrystals for use as highly efficient MRI contrast agent. $J$. Mater. Chem. 2011, 21 (31), 11472-11477.

77. Agthe, M.; Wetterskog, E.; Mouzon, J.; SalazarAlvarez, G.; Bergström, L., Dynamic growth modes of ordered arrays and mesocrystals during drop-casting of iron oxide nanocubes. CrystEngComm 2014, 16 (8), 1443-1450.

78. Schuck, P. P., SEDFIT version 16.1c. http://analyticalultracentrifugation.com/download.htm.

79. Schuck, P.; Rossmanith, P., Determination of the sedimentation coefficient distribution by least-squares boundary modeling. Biopolymers 2000, 54 (5), 328-41.

80. Demeler, B., UltraScan version 4.0, release 2783. A Comprehensive Data Analysis Software Package for Analytical Ultracentrifugation Experiments. The University of Lethbridge, Department of Chemistry and Biochemistry. http://www.ultrascan3.aucsolutions.com/download.php.

81. Brookes, E.; Cao, W.; Demeler, B., A two-dimensional spectrum analysis for sedimentation velocity experiments of mixtures with heterogeneity in molecular weight and shape. Eur Biophys J 2010, 39 (3), 405-14.

82. Demeler, B.; Nguyen, T. L.; Gorbet, G. E.; Schirf, V.; Brookes, E. H.; Mulvaney, P.; El-Ballouli, A. O.; Pan, J.; Bakr, O. M.; Demeler, A. K.; Hernandez Uribe, B. I.; Bhattarai, N.; Whetten, R. L., Characterization of size, anisotropy, and density heterogeneity of nanoparticles by sedimentation velocity. Anal. Chem. 2014, 86 (15), 7688-95.

83. Filik, J.; Ashton, A. W.; Chang, P. C. Y.; Chater, P. A.; Day, S. J.; Drakopoulos, M.; Gerring, M. W.; Hart, M. L.; Magdysyuk, O. V.; Michalik, S.; Smith, A.; Tang, C. C.; Terrill, N. J.; Wharmby, M. T.; Wilhelm, H., Processing two-dimensional $\mathrm{X}$-ray diffraction and small-angle scattering data in DAWN 2. $J$. Appl. Crystallogr. 2017, 50 (Pt 3), 959-966.

84. Yang, X.; Juhas, P.; Farrow, C. L.; Billinge, S. J. L., xPDFsuite: an end-to-end software solution for high throughput pair distribution function transformation, visualization and analysis. J. Appl. Crystallogr. 2013. 


\section{SYNOPSIS TOC}

This study describes the synthesis and morphogenetic aspects of faceted magnetite mesocrystals. The assembly of nanoparticles is affected by solvent and non-solvent nature, nanoparticle and surfactant concentration as well as by nanoparticle size, shape, and solvation shell. The observed changes of mesocrystal structure are to a certain extent in analogy to structural polymorphism known for "classical" crystals.

\section{Table of Contents artwork}

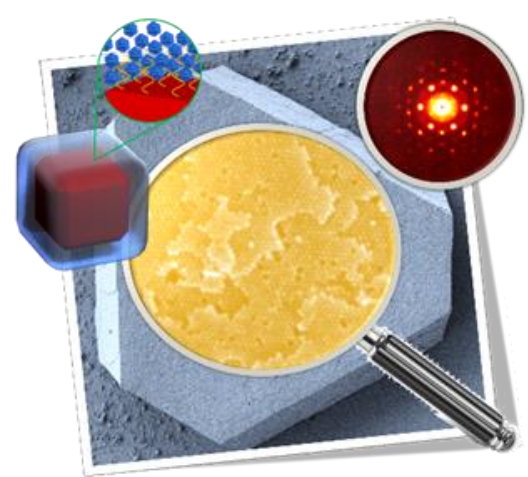

